\title{
Special Vehicle Oil-Gas Suspension System Design and Implementation
}

\author{
Li Ning ${ }^{1, a}$, Jiang Wei ${ }^{1, b}$, Tao $\mathrm{Yi}^{2, \mathrm{c}}$, Zhao Shan-liang ${ }^{1}$, Ren Gang ${ }^{3}$ \\ ${ }^{1}$ Wuhan Mechanical Technology College, Wuhan, China \\ ${ }^{2}$ Beijing Special Vehicle Academy, Beijing, China \\ ${ }^{3}$ The $95963^{\text {th }}$ Troops of PLA, Wuhan, China \\ ajw007305@126.com, bjw007305@163.com, ctaotaoyiyi@163.com
}

Keywords: special vehicle; oil and gas suspension; test platform; design and implementation

\begin{abstract}
The oil and gas suspension is the important part of the type special vehicle. This paper introduces the working principle of the special type vehicle oil gas suspension system, and the multifunctional experiment debugging platform is designed based on PROE modeling technology, and it is used to inspect the characteristics of the oil and gas suspension system. The overall design ideas of the platform, the test schematic diagrams of the test parts and the platform prototype structure are described. The platform can provide a new test methods and means for the vehicle maintenance personnel.
\end{abstract}

\section{Introduction}

The oil and gas suspension system is the new passive suspension system that includes the damper and the spring element on seventy years or so. It has the functions of the damper and the spring element, and transfers pressure by the oil, and the high pressure gas is used as the elastic medium. It can meet the requirements of the engineering vehicles with its superior nonlinear elastic properties and good vibration reduction performance. Compared with the traditional suspension including the steel plate spring and the cylinder type shock absorber, the oil and gas suspension system has many advantages, such as the nonlinear elastic property, the height of the body that can free adjustment, the small volume, the light weight, the rigid lock that can be achieved and so on. Because of the many advantages of the oil and gas suspension system, it has been widely applied to the engineering vehicle and the special vehicle [1].

\section{The structure and the working principle of the oil and gas suspension system}

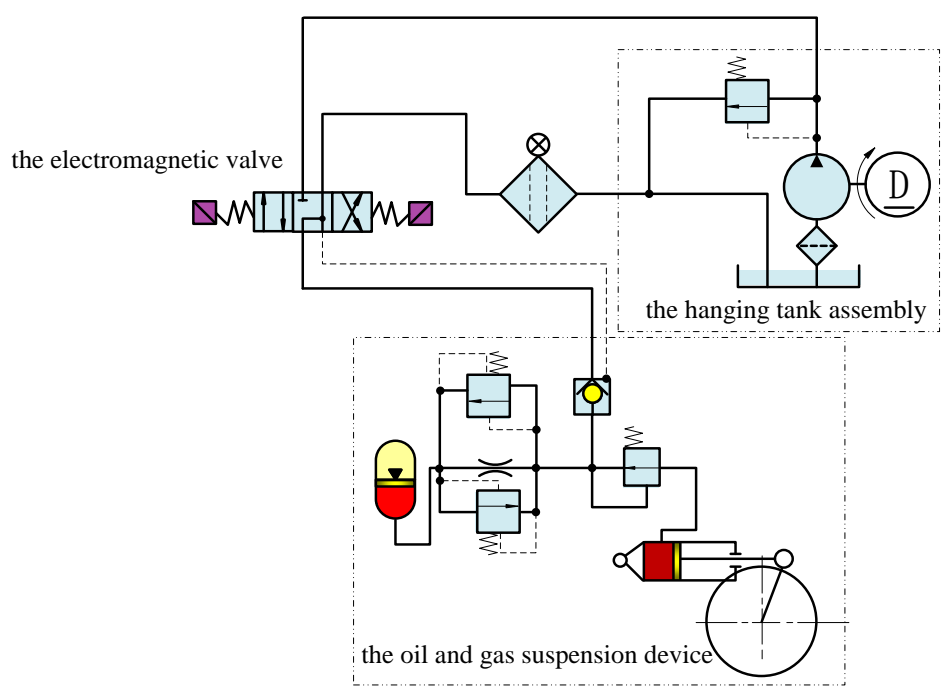

Figure 1 the working principle diagram of the oil and gas suspension system of the special vehicle

The working principle diagram of the oil and gas suspension system of the special vehicle in Figure 1, and is mainly composed of the hanging tank assembly, the electromagnetic valve group, 
the oil and gas suspension device (the power cylinder and the accumulator) and so on.

\section{Buffer}

When the vehicle runs in a raised obstacle, the wheels are impacted and rise to the body, and the balance elbow rotates, and the inner cylinder of the power cylinder moves upward. The high pressure oil flows into the shock pressure storage device to push the floating piston, and the floating piston compresses the nitrogen, and some energy is stored, and this eases the impact of the body; at the same time, the torsion of the torsion shaft also absorbs the impact energy, and thus the impact of the chassis is eased [2].

\section{Shock-absorbing}

After the vehicle runs in a raised obstacle, the balance elbow rotates the torsion shaft, and the compressed nitrogen expanses and releases the absorbed energy, and the inner cylinder of the power cylinder extends and makes the balance elbow down swing, and the wheel presses the caterpillar track, and this keeps the caterpillar track and the ground with the good adhesion. While the Nitrogen is compressed and expanded, the high-pressure oil highly flows between the power cylinder and accumulator device, and generates the damping through the pipe, pipe fittings and so on, and the part of the vibration energy is absorbed. Therefore, the vehicle vibration is decayed very quickly.

\section{Adjusting the height of the car body}

The pressure oil flowing in and out of the damping pressure storage device and the power cylinder oil chamber are controlled through the electromagnetic valve, and the chamber pressure of compressed gas is the same as the oil filled pressure [3]; The inner cylinder of the power cylinder is extended and retracted by filling in and out of the power cylinder oil, and the balance elbow is swung by pulling the arm, and so the vehicle body is increased or decreased. When the bottom roses to the high, stop feeding, and the charge / discharge tube is connected to the oil tank, and the steel ball returns immediately under the effect of the spring and the oil pressure, and the oil pressure and the gas pressure are in the equilibrium [4].

\section{Design of the debug platform}

\section{The overall design ideas}

The debug platform is mainly used for the test of the main static and dynamic performance of the oil and gas suspension system, and includes the test circuit of the suspension tank and the device design, the test circuit of the electromagnetic valve and the device design, the test circuit of the suspension dynamic load and the loading device design and so on, and the completion of the test control relationship is as shown in Figure 2.

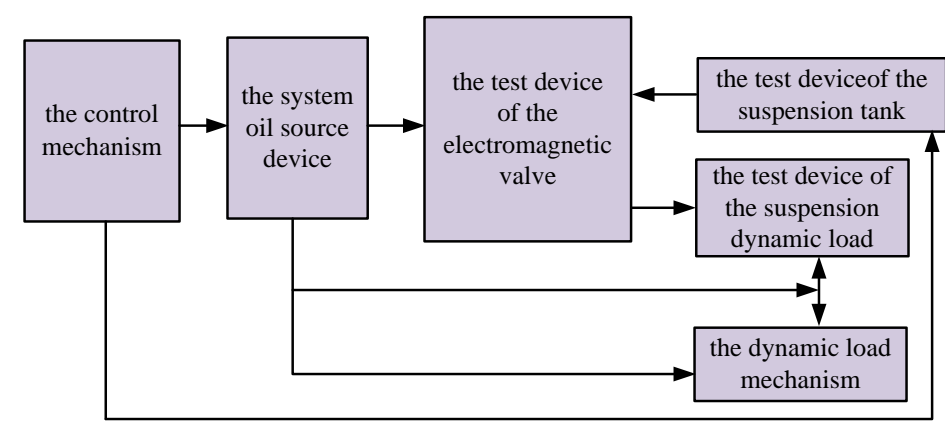

Figure 2 the test control diagram of the debug platform

\section{The test circuit design of the suspension fuel tank}

The suspension fuel tank is the power source of the suspension device, and but also the control mechanism of the system pressure, and mainly includes the tank, the direct-current motor, the gear pump, the pressure regulating valve, the return check valve and so on. According to its working characteristics, the test is done to the reliability of the motor, the gear pump efficiency and the static and dynamic characteristics of the overflow valve, and the test circuit is shown in figure 3. 


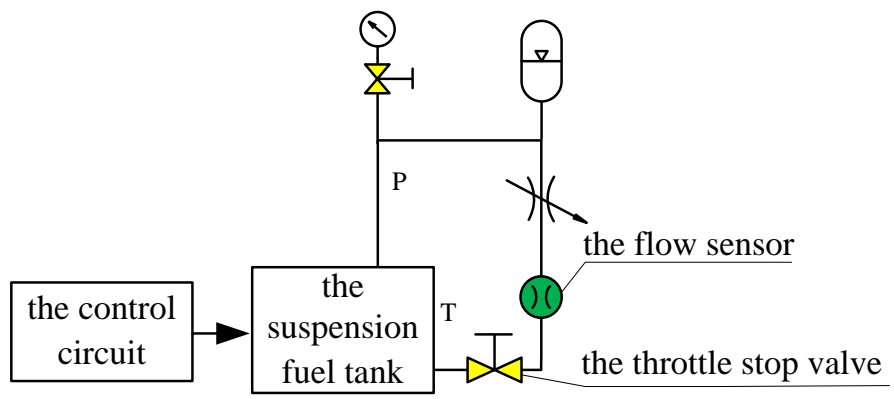

Figure 3 the schematic diagram of the test circuit for the suspension fuel tank

\section{The test circuit design of the solenoid valve}

The solenoid valve is used to control the access of the track adjuster and the oil circuit of the suspension mechanism, and consists of five valves, and the inlet and outlet pipe is shared, and according to the characteristics of each electromagnetic valve, the electrical characteristics, the reversing and the leakage are tested. The test circuit is shown in Figure 4.

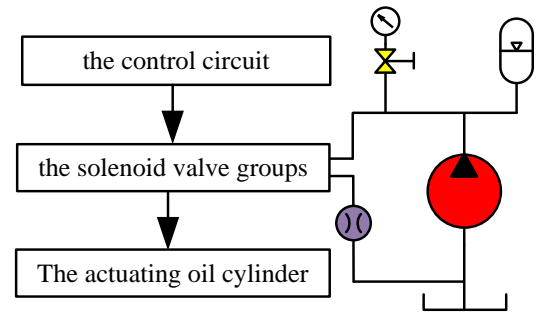

Figure 4 the test circuit design diagram of the solenoid valve

\section{The dynamic load test circuit design of the suspension device}

The suspension device mainly consists of the power cylinder and the accumulator, and is linked with the balance elbow and the loading wheel by the power cylinder, and has the function of the cushioning, the shock and adjusting the vehicle pose. The action of the telescopic performance and the sealing performance of the power cylinder under the variable load and the storage storing the pressure performance and the sealing property are tested, and the test circuit design principle is shown in Figure 5.



Figure 5 the schematic diagram of the dynamic load test circuit for the suspension device

\section{The design of the loading oil source}

The loading oil source is mainly used to supply the pressure and the flow for the test of the suspension dynamic load, and but also is the hydraulic source used to do the test of the components under the conditions without hanging tank, and its structure and working principle are shown in Figure 6. 


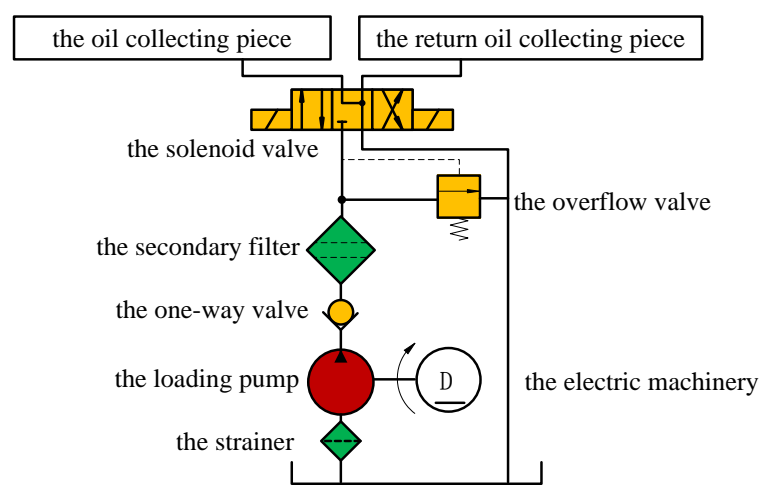

Figure 6 the design schematic of the system oil source

\section{The realization of the platform prototype}

The realization of the debugging platform is developed based on PROE software and produced and tested, and it is mainly composed of the test platform, the hanging tank testing fixture, the electromagnetic valve test fixture, the suspension device test fixture, the loading oil source, the shield support, the console, the electrical control parts and so on. It is shown in Figure 7.

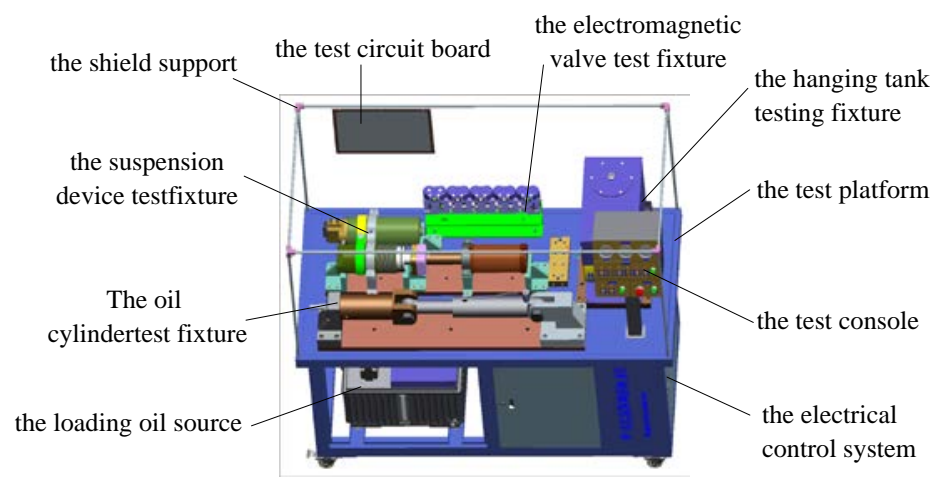

Figure 7 the composition diagram of the debugging platform

Through the application of the platform, the test results are achieved. The debugging platform has the following characteristics:

(1) The strong function and the strong extension. The platform can not only complete the test oil and gas suspension system function of two different special equipment chassis, and but also can connected all parts and reproduce the structure of the suspension system, and can achieve the function simulation training, and can also carry out the performance test of other similar components.

(2) The design is novel, the structure is reasonable. The oil and gas suspension debugging platform of the special vehicle is the first design in an industry, and the platform modeling is designed through the PROE software, and the assembly layout is optimized combined test piece model, and the strength company does processing and assembling, and the test module distribution of the institutions is reasonable, and it is the reliable installation.

(3) It runs smoothly, and has the high automation degree. The sensor technology and PLC control technology are applied in the platform, and so that the platform runs smoothly and the high automation degree, and the reliability and accuracy of the test are greatly improved.

\section{Conclusion}

By analysis of the principle of the suspension system, and the PROE modeling, sensor technology and PLC control technology are applied, and the debugging platform is designed and implemented, and it has been applied in the teaching process, and the desired development is achieved, and it enriches the vehicle maintenance support means, and it has the good economic value and the application prospect. 


\section{References}

[1] Wu Ren-zhi. Simulation and experiment study on dynamics model of HPs system[D]. Hangzhou: College of Mechanical and Energy Engineering, Zhejiang University, 2000.

[2] Ma Guo-qing, Wang Shu-xin, Tan Run-hua.Study on the characteristics of hydropneumatic suspensionsystem[J].chinese Hydraulics \& Pneumatics, 2003(6): 978-981.

[3] Ma Guo-qing, Tan Run-hua. Nonlinear mathematic model of hydropenumatic suspension in crane vehicles and it's simulation[J].Chinese Journal of Mechanical Engineering, 2002, 38(5): 7l-74.

[4] Feng Shi-cai, Wu Ren-zhi. Test and research in oil-gas suspension performance of construction vehicles[J].Construction Machinery and Equipment, 2000(12): 9-11. 